\title{
Antibiofilm efficacy of nitric oxide-releasing alginates against cystic fibrosis bacterial pathogens
}

\author{
Mona Jasmine R. Ahonen, ${ }^{\dagger}$ Jamie M. Dorrier, ${ }^{\dagger}$ and Mark H. Schoenfisch ${ }^{\dagger+*}$ \\ 'Department of Chemistry, University of North Carolina at Chapel Hill, CB 3290, Chapel Hill, \\ NC 27599, United States \\ Division of Pharmacoengineering and Molecular Pharmaceutics, UNC Eshelman School of \\ Pharmacy, Chapel Hill, NC 27599
}

*To whom correspondence should be addressed: schoenfisch@unc.edu

\section{Table of Contents}

Table S1. Molecular weight and nitrogen content of unmodified- and alkyl amine-modified alginates.

Figure S1. Representative ${ }^{13} \mathrm{C}$ NMR alkyl amine-modified alginates.

List of representative 1H and 13C NMR peals for unmodified and alkyl

amine-modified alginates.

Table S2. Nitric oxide-release in phosphate buffered saline

(PBS, $\left.\mathrm{pH} 6.5,37^{\circ} \mathrm{C}\right)$

Figure S2. Representative UV-vis spectra for secondary-amine and $N$-diazeniumdiolate alginate.

Figure S3. Real-time NO-release profiles and total NO release

of NO-releasing alginates in artificial sputum media (ASM).

Figure S4. Anti-biofilm efficacy of control alginates against $S$. aureus

biofilms under (A) aerobic and (B) anaerobic conditions 
Table S1. Average molecular weight, polydispersity, and nitrogen content of bare and alkyl amine-modified alginates. ${ }^{a}$

\begin{tabular}{llll}
\hline Biopolymers & ${\text { Average Molecular Weight }(\mathbf{D a})^{\boldsymbol{b}}}$ & $\mathbf{P D I}^{\boldsymbol{b}}$ & $\mathbf{N}(\boldsymbol{\%})^{\boldsymbol{c}}$ \\
\hline Alg5 & 4,680 & 1.20 & $0.0 \pm 0.0$ \\
Alg5-DETA & 5,200 & 2.10 & $7.8 \pm 0.8$ \\
Alg5-DPTA & 6,000 & 1.40 & $6.4 \pm 0.2$ \\
Alg5-SPER & 7,900 & 1.80 & $8.2 \pm 1.1$ \\
Alg5-PAPA & 6,700 & 1.50 & $5.7 \pm 0.7$ \\
Alg5-PAPA-DPTA & 7,200 & 1.90 & $11.2 \pm 0.7$ \\
\hline
\end{tabular}

${ }^{a}$ Error represents standard deviation for $\mathrm{n} \geq 3$ experiments. ${ }^{b}$ Measured in using a gel permeation chromatography (GCP)-multiangle light scattering system. ${ }^{c}$ Measured by elemental analysis.

A

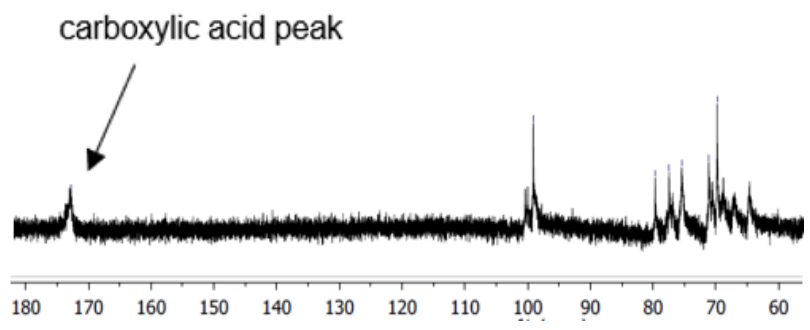

B

carboxylic acid peak



Figure S1. (A) Representative ${ }^{1} \mathrm{C}$ NMR spectrum for (A) Alg5, and (B) Alg5-PAPA-DPTA in $\mathrm{D}_{2} \mathrm{O}$. 


\section{List of representative ${ }^{1} \mathrm{H}$ and ${ }^{13} \mathrm{C}$ NMR peaks for unmodified and alkyl amine-modified alginates}

Alg5: ${ }^{1} \mathrm{H}$ NMR $\left(600 \mathrm{MHz}, \mathrm{D}_{2} \mathrm{O}, \delta\right)$ 3.60-4.05 $(\mathrm{OCHCH}(\mathrm{OH}) \mathrm{CH}(\mathrm{OH})), 4.30(\mathrm{OCHCH}(\mathrm{OH}) \mathrm{CH}(\mathrm{OH})$, 4.50-4.60 (CHCOOH), $4.90 \quad(\mathrm{OCH}(\mathrm{CHOH}) \mathrm{O}) .{ }^{13} \mathrm{C} \quad \mathrm{NMR} \quad\left(\begin{array}{llllll}600 & \left.\mathrm{MHz}, \quad \mathrm{D}_{2} \mathrm{O}, \quad \delta\right) & 65.0-80.0\end{array}\right.$ $(\mathrm{OCHCH}(\mathrm{OH}) \mathrm{CH}(\mathrm{OH}) C \mathrm{H}(\mathrm{OH}) C \mathrm{H}(\mathrm{O})), 100.0(\mathrm{OCHCH}(\mathrm{OH})), 175.0(\mathrm{CHC}(\mathrm{O}))$.

Alg5-DPTA: ${ }^{1} \mathrm{H}$ NMR (600 MHz, $\mathrm{D}_{2} \mathrm{O}, \delta$ ) 1.60-1.80 $\left(\mathrm{CH}_{2} \mathrm{CH}_{2} \mathrm{CH}_{2} \mathrm{NHCH}_{2} \mathrm{CH}_{2} \mathrm{CH}_{2} \mathrm{NH}_{2}\right), \quad 2.60-2.30$ $\left(\mathrm{CH}_{2} \mathrm{CH}_{2} \mathrm{CH}_{2} \mathrm{NHCH}_{2} \mathrm{CH}_{2} \mathrm{CH}_{2} \mathrm{NH}_{2}\right), \quad 2.80-3.10 \quad\left(\mathrm{CH}_{2} \mathrm{CH}_{2} \mathrm{CH}_{2} \mathrm{NHCH}_{2} \mathrm{CH}_{2} \mathrm{CH}_{2} \mathrm{NH}_{2}\right), \quad 3.60-4.05$ $(\mathrm{OCHCH}(\mathrm{OH}) \mathrm{CH}(\mathrm{OH})), 4.30(\mathrm{OCHCH}(\mathrm{OH}) \mathrm{CH}(\mathrm{OH}), 4.50-4.60(\mathrm{NHCOCH}), 4.90(\mathrm{OCH}(\mathrm{CHOH}) \mathrm{O})$. ${ }^{13} \mathrm{C} \mathrm{NMR}\left(600 \mathrm{MHz}, \mathrm{D}_{2} \mathrm{O}, \delta\right)$ 26.9-29.5 (C(O) $\left.\mathrm{NHCH}_{2} \mathrm{CH}_{2} \mathrm{CH}_{2} \mathrm{NH}, \mathrm{NHCH}_{2} \mathrm{CH}_{2} \mathrm{CH}_{2} \mathrm{NH}_{2}\right)$, 37.6-46.0 CH2 $\left(\mathrm{C}(\mathrm{O}) \mathrm{NHCH}_{2} \mathrm{CH}_{2} \mathrm{CH}_{2} \mathrm{NH}, \quad \mathrm{NHCH}_{2} \mathrm{CH}_{2} \mathrm{CH}_{2} \mathrm{NH}_{2}\right), \quad 65.0-80.0(\mathrm{OCHCH}(\mathrm{OH}) \mathrm{CH}(\mathrm{OH}) \mathrm{CH}(\mathrm{OH}) \mathrm{CH}(\mathrm{O}))$, $100.0(\mathrm{OCHCH}(\mathrm{OH})), 160.0(\mathrm{CHC}(\mathrm{O}) \mathrm{NH}), 175.0(\mathrm{CHC}(\mathrm{O}))$.

Alg5-DETA: ${ }^{1} \mathrm{H} \quad \mathrm{NMR} \quad\left(600 \mathrm{MHz}, \quad \mathrm{D}_{2} \mathrm{O}, \quad \delta\right) \quad 2.30-3.30 \quad\left(\mathrm{CH}_{2} \mathrm{CH}_{2} \mathrm{NHCH}_{2} \mathrm{CH}_{2} \mathrm{NH}_{2}\right), \quad 3.60-4.05$ $(\mathrm{OCHCH}(\mathrm{OH}) \mathrm{CH}(\mathrm{OH})), 4.30(\mathrm{OCHCH}(\mathrm{OH}) \mathrm{CH}(\mathrm{OH}), 4.50-4.60(\mathrm{NHCOCH}), 4.90(\mathrm{OCH}(\mathrm{CHOH}) \mathrm{O})$. ${ }^{13} \mathrm{C} \quad \mathrm{NMR} \quad\left(600 \quad \mathrm{MHz}, \quad \mathrm{D}_{2} \mathrm{O}, \quad \delta\right) \quad 39.0-47.0 \quad\left(\mathrm{C}(\mathrm{O}) \mathrm{NHCH}_{2} \mathrm{CH}_{2} \mathrm{NHCH}_{2} \mathrm{CH}_{2} \mathrm{NH}_{2}\right), \quad 65.0-80.0$ $(\mathrm{OCHCH}(\mathrm{OH}) \mathrm{CH}(\mathrm{OH}) \mathrm{CH}(\mathrm{OH}) \mathrm{CH}(\mathrm{O})), 100.0(\mathrm{OCHCH}(\mathrm{OH})), 160.0(\mathrm{CHC}(\mathrm{O}) \mathrm{NH}), 175.0(\mathrm{CHC}(\mathrm{O}))$.

Alg5-PAPA: ${ }^{1} \mathrm{H}$ NMR (600 MHz, $\left.\mathrm{D}_{2} \mathrm{O}, \delta\right)$ 0.70-0.80 $\left(\mathrm{NHCH}_{2} \mathrm{CH}_{2} \mathrm{CH}_{3}\right), 1.52\left(\mathrm{NHCH}_{2} \mathrm{CH}_{2} \mathrm{CH}_{3}\right), 1.85$ $\left(\mathrm{CH}_{2} \mathrm{CH}_{2} \mathrm{CH}_{2} \mathrm{NHCH}_{2} \mathrm{CH}_{2} \mathrm{CH}_{3}\right), \quad 2.80-3.10 \quad\left(\mathrm{CH}_{2} \mathrm{CH}_{2} \mathrm{CH}_{2} \mathrm{NHCH}_{2} \mathrm{CH}_{2} \mathrm{CH}_{3}\right), \quad 3.60-4.05$ $(\mathrm{OCHCH}(\mathrm{OH}) \mathrm{CH}(\mathrm{OH})), 4.30(\mathrm{OCHCH}(\mathrm{OH}) \mathrm{CH}(\mathrm{OH}), 4.50-4.60(\mathrm{NHCOCH}), 4.90(\mathrm{OCH}(\mathrm{CHOH}) \mathrm{O})$. ${ }^{13} \mathrm{C} \quad \mathrm{NMR} \quad\left(600 \mathrm{MHz}, \quad \mathrm{D}_{2} \mathrm{O}, \quad \delta\right) \quad 10.9 \quad\left(\mathrm{NHCH}_{2} \mathrm{CH}_{2} \mathrm{CH}_{3}\right), \quad 20.0 \quad\left(\mathrm{NHCH}_{2} \mathrm{CH}_{2} \mathrm{CH}_{3}\right), \quad 31.3-49.0$ $\left(\mathrm{C}(\mathrm{O}) \mathrm{NHCH}_{2} \mathrm{CH}_{2} \mathrm{CH}_{2} \mathrm{NH}, \mathrm{NHCH}_{2} \mathrm{CH}_{2} \mathrm{CH}_{3}\right), 65.0-80.0(\mathrm{OCHCH}(\mathrm{OH}) \mathrm{CH}(\mathrm{OH}) \mathrm{CH}(\mathrm{OH}) \mathrm{CH}(\mathrm{O})), 100.0$ $(\mathrm{OCHCH}(\mathrm{OH})), 160.0(\mathrm{CHC}(\mathrm{O}) \mathrm{NH}), 175.0(\mathrm{CHC}(\mathrm{O}))$.

Alg5-SPER: ${ }^{1} \mathrm{H}$ NMR $\left(600 \mathrm{MHz}, \mathrm{D}_{2} \mathrm{O}, \delta\right) 1.13\left(\mathrm{NHCH}_{2}\left(\mathrm{CH}_{2}\right)_{2} \mathrm{CH}_{2} \mathrm{NH}\right), 1.56\left(\mathrm{NHCH}_{2} \mathrm{CH}_{2} \mathrm{CH}_{2} \mathrm{NH}\right), 1.80$ $\left(\mathrm{C}(\mathrm{O}) \mathrm{NHCH}_{2} \mathrm{CH}_{2} \mathrm{CH}_{2} \mathrm{NH}\right), 2.20-2.40\left(\mathrm{CH}_{2} \mathrm{CH}_{2} \mathrm{CH}_{2} \mathrm{NH}, \mathrm{NHCH}_{2}\left(\mathrm{CH}_{2}\right)_{2} \mathrm{CH}_{2} \mathrm{NH}, \mathrm{NHCH}_{2} \mathrm{CH}_{2} \mathrm{CH}_{2} \mathrm{NH}_{2}\right)$, $2.68\left(\mathrm{NHCH}_{2} \mathrm{CH}_{2} \mathrm{CH}_{2} \mathrm{NH}_{2}\right), \quad 2.80-3.10\left(\mathrm{C}(\mathrm{O}) \mathrm{NHCH}_{2} \mathrm{CH}_{2} \mathrm{CH}_{2} \mathrm{NH}, \quad \mathrm{NHCH}_{2} \mathrm{CH}_{2} \mathrm{CH}_{2} \mathrm{NH}_{2}\right), \quad 3.60-4.05$ $(\mathrm{OCHCH}(\mathrm{OH}) \mathrm{CH}(\mathrm{OH})), 4.30(\mathrm{OCHCH}(\mathrm{OH}) \mathrm{CH}(\mathrm{OH}), 4.50-4.60(\mathrm{NHCOCH}), 4.90(\mathrm{OCH}(\mathrm{CHOH}) \mathrm{O})$. ${ }^{13} \mathrm{C}$ NMR $\left(600 \mathrm{MHz}, \mathrm{D}_{2} \mathrm{O}, \delta\right) 23.2\left(\mathrm{NHCH}_{2}\left(\mathrm{CH}_{2}\right)_{2} \mathrm{CH}_{2} \mathrm{NH}\right), 34.8-45.0\left(\mathrm{C}(\mathrm{O}) \mathrm{NHCH}_{2} \mathrm{CH}_{2} \mathrm{CH}_{2} \mathrm{NH}\right), 65.0-$ $80.0(\mathrm{OCHCH}(\mathrm{OH}) \mathrm{CH}(\mathrm{OH}) \mathrm{CH}(\mathrm{OH}) \mathrm{CH}(\mathrm{O})), \quad 100.0 \quad(\mathrm{OCHCH}(\mathrm{OH})), \quad 160.0 \quad(\mathrm{CHC}(\mathrm{O}) \mathrm{NH}), \quad 175.0$ $(\mathrm{CHC}(\mathrm{O}))$.

Alg5-PAPA-DPTA: ${ }^{1} \mathrm{H}$ NMR (600 MHz, $\left.\mathrm{D}_{2} \mathrm{O}, \delta\right)$ 0.70-0.80 $\left(\mathrm{NHCH}_{2} \mathrm{CH}_{2} \mathrm{CH}_{3}\right), 1.48\left(\mathrm{NHCH}_{2} \mathrm{CH}_{2} \mathrm{CH}_{3}\right)$, 1.50-1.70 ( $\left.\mathrm{CH}_{2} \mathrm{CH}_{2} \mathrm{CH}_{2} \mathrm{NHCH}_{2} \mathrm{CH}_{2} \mathrm{CH}_{2} \mathrm{NH}_{2}\right), \quad 2.30-2.40 \quad\left(\mathrm{CH}_{2} \mathrm{CH}_{2} \mathrm{CH}_{2} \mathrm{NHCH}_{2} \mathrm{CH}_{2} \mathrm{CH}_{2} \mathrm{NH}_{2}\right), \quad 2.10$ $\left(\mathrm{CH}_{2} \mathrm{CH}_{2} \mathrm{CH}_{2} \mathrm{NHCH}_{2} \mathrm{CH}_{2} \mathrm{CH}_{3}\right), \quad 2.60-3.10 \quad\left(\mathrm{CH}_{2} \mathrm{CH}_{2} \mathrm{CH}_{2} \mathrm{NHCH}_{2} \mathrm{CH}_{2} \mathrm{CH}_{2} \mathrm{NH}_{2}\right), \quad 3.60-4.05$ $(\mathrm{OCHCH}(\mathrm{OH}) \mathrm{CH}(\mathrm{OH})), 4.30(\mathrm{OCHCH}(\mathrm{OH}) \mathrm{CH}(\mathrm{OH}), 4.50-4.60(\mathrm{NHCOCH}), 4.90(\mathrm{OCH}(\mathrm{CHOH}) \mathrm{O})$. ${ }^{13} \mathrm{C} \quad \mathrm{NMR} \quad\left(600 \mathrm{MHz}, \quad \mathrm{D}_{2} \mathrm{O}, \quad \delta\right) \quad 9.4 \quad\left(\mathrm{NHCH}_{2} \mathrm{CH}_{2} \mathrm{CH}_{3}\right), \quad 20.0 \quad\left(\mathrm{NHCH}_{2} \mathrm{CH}_{2} \mathrm{CH}_{3}\right), \quad 26.9-29.5$ $\left(\mathrm{C}(\mathrm{O}) \mathrm{NHCH}_{2} \mathrm{CH}_{2} \mathrm{CH}_{2} \mathrm{NH}, \quad \mathrm{NHCH}_{2} \mathrm{CH}_{2} \mathrm{CH}_{2} \mathrm{NH}_{2}\right), \quad 31.3-49.0 \quad\left(\mathrm{C}(\mathrm{O}) \mathrm{NHCH}_{2} \mathrm{CH}_{2} \mathrm{CH}_{2} \mathrm{NH}\right.$, $\left.\mathrm{NHCH}_{2} \mathrm{CH}_{2} \mathrm{CH}_{3}\right), \quad 37.6-46.0 \quad \mathrm{CH} 2 \quad\left(\mathrm{C}(\mathrm{O}) \mathrm{NHCH}_{2} \mathrm{CH}_{2} \mathrm{CH}_{2} \mathrm{NH}, \quad \mathrm{NHCH}_{2} \mathrm{CH}_{2} \mathrm{CH}_{2} \mathrm{NH}_{2}\right), \quad$ 65.0-80.0 $(\mathrm{OCHCH}(\mathrm{OH}) \mathrm{CH}(\mathrm{OH}) \mathrm{CH}(\mathrm{OH}) \mathrm{CH}(\mathrm{O})), 100.0(\mathrm{OCHCH}(\mathrm{OH})), 160.0(\mathrm{CHC}(\mathrm{O}) \mathrm{NH}), 175.0(\mathrm{CHC}(\mathrm{O}))$ 
Table S2. Nitric oxide-release properties of $N$-diazeniumdiolate-functionalized alginates in PBS (pH $\left.6.5,37^{\circ} \mathrm{C}\right){ }^{a}$

\begin{tabular}{lllll}
\hline Biopolymer & $\begin{array}{l}\mathbf{t}[\mathbf{N O}]^{b} \\
(\boldsymbol{\mu m o l} / \mathbf{m g})\end{array}$ & $\begin{array}{l}{[\mathbf{N O}]_{\max }{ }^{\boldsymbol{c}}} \\
(\mathbf{p p b} / \mathbf{m g})\end{array}$ & $\boldsymbol{t}_{\mathbf{1 / 2}}{ }^{\boldsymbol{d}}(\mathbf{m i n s})$ & $\boldsymbol{t}_{\mathbf{d}}{ }^{\boldsymbol{e}}(\mathbf{h})$ \\
\hline Alg5-DETA/NO & $0.36 \pm 0.10$ & $590 \pm 20$ & $144 \pm 84$ & $16.0 \pm 3.9$ \\
Alg5-DPTA/NO & $0.42 \pm 0.03$ & $1000 \pm 230$ & $24 \pm 7$ & $4.5 \pm 0.2$ \\
Alg5-SPER/NO & $0.37 \pm 0.06$ & $2890 \pm 330$ & $18 \pm 6$ & $3.3 \pm 0.9$ \\
Alg5-PAPA/NO & $0.60 \pm 0.03$ & $12100 \pm 3800$ & $6 \pm 3$ & $2.7 \pm 0.1$ \\
Alg5-PAPA-DPTA/NO & $0.55 \pm 0.04$ & $4890 \pm 1300$ & $21 \pm 5$ & $4.8 \pm 1.4$ \\
\hline
\end{tabular}

${ }^{a}$ Error represents standard deviation for $\mathrm{n} \geq 3$ experiments. ${ }^{b}$ Total NO released. ${ }^{c}$ Maximum flux of NO release. ${ }^{d}$ NO-release half-life. ${ }^{e}$ Duration of NO release. 

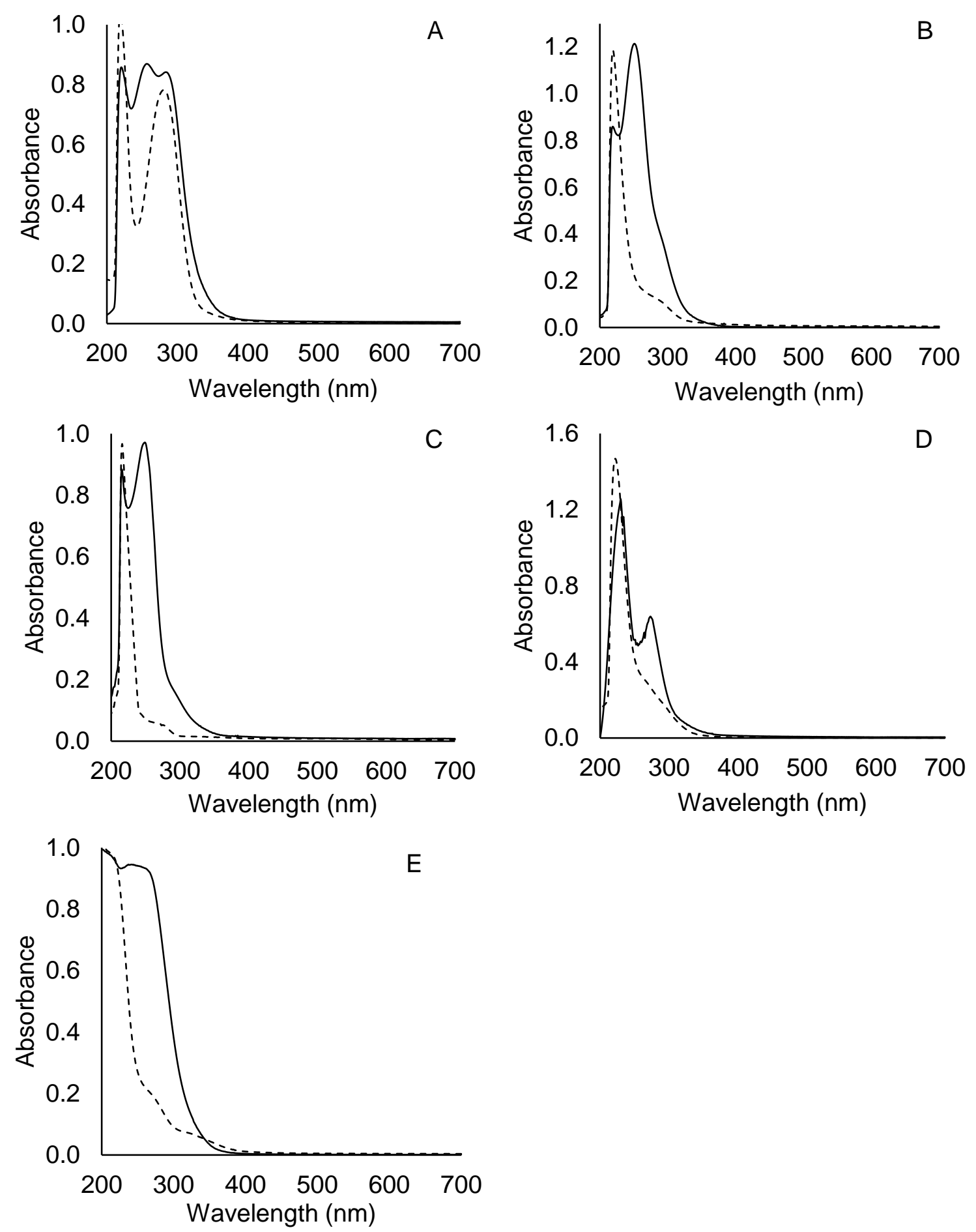

Figure S2. Representative UV-vis spectra for secondary amine- (dash) and $\mathrm{N}$ diazeniumdiolate-modified (solid) (A) Alg5-DETA, (B) Alg5-DPTA, (C) Alg5-SPER, (D), Alg5-PAPA, and (E) Alg5-PAPA-DPTA. 

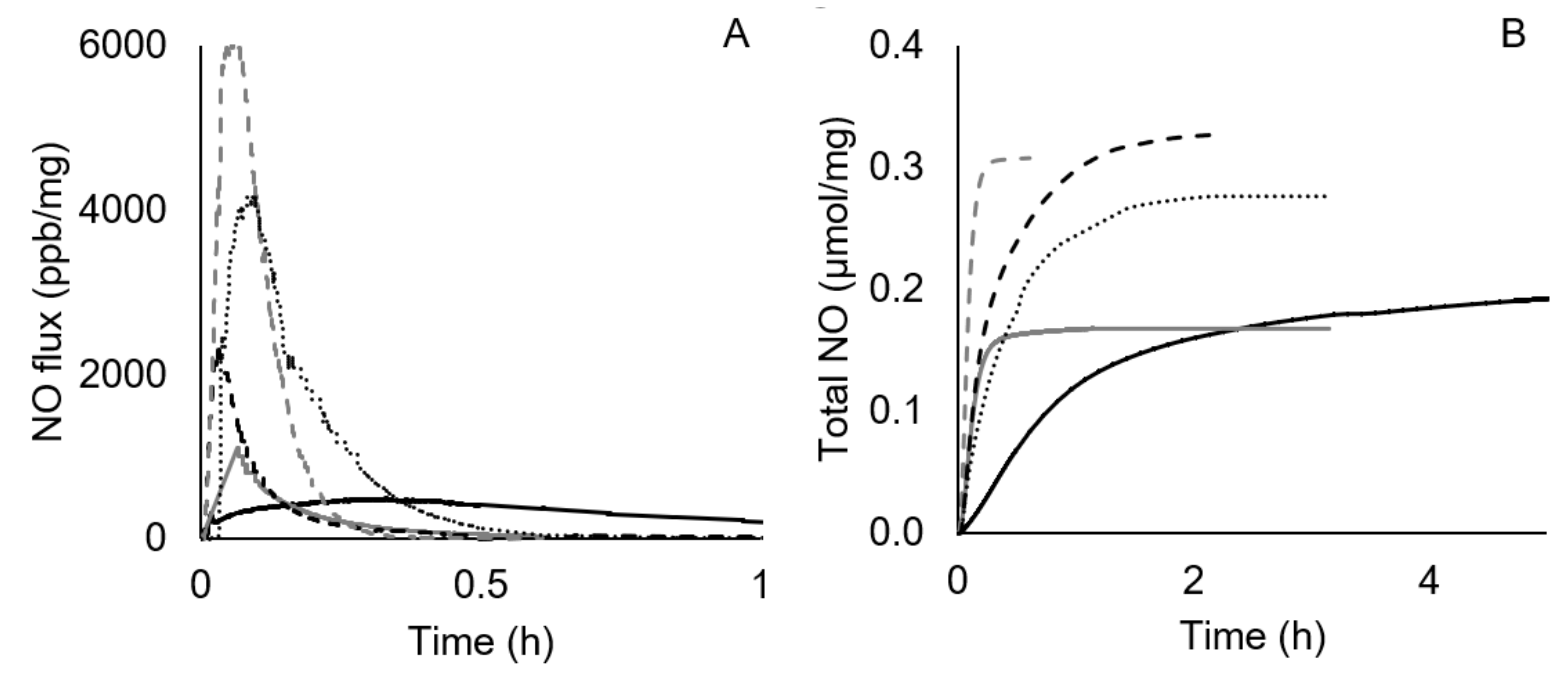

Figure S3. (A) Real-time NO-release profiles for the first $1 \mathrm{~h}$ and (B) plot of total NO release vs. time for representative biopolymers Alg5-DETA/NO (black solid), Alg5-DPTA/NO (gray solid), Alg5-SPER/NO (black dash), Alg5-PAPA/NO (gray dash), and Alg5-PAPADPTA/NO (black dot) measured via chemiluminescence in ASM pH 6.5. 

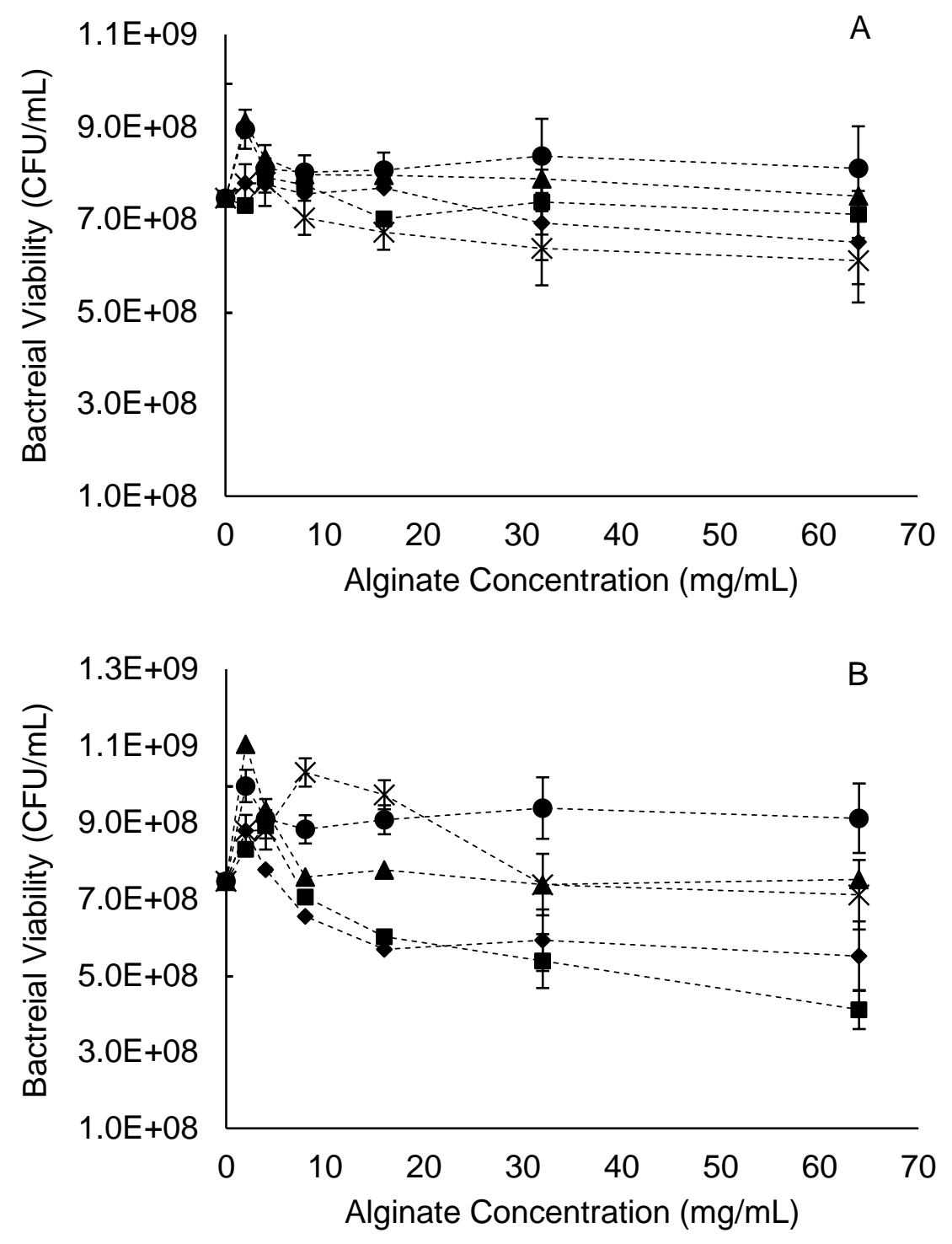

Figure S4. Anti-biofilm efficacy of Alg5-DETA (circle), Alg5-DPTA (triangle), Alg5-SPER (square), Alg5-PAPA (diamond), and Alg5-PAPA-DPTA (cross) against S. aureus biofilms under (A) aerobic and (B) anaerobic conditions. Error bars represent standard deviation of the mean viability $(\mathrm{CFU} / \mathrm{mL})$. For all measurements, $\mathrm{n} \geq 3$ pooled experiments. 